\title{
High Circulating Alarin Levels Are Associated with Presence of Metabolic Syndrome
}

\author{
Xia Fang ${ }^{\mathrm{a}}$ Tingran Zhang ${ }^{\mathrm{b}}$ Mengliu Yang ${ }^{\mathrm{a}, \mathrm{c}} \quad$ Ling Li $^{\mathrm{a}} \quad$ Cheng Zhang ${ }^{\mathrm{a}}$ \\ Wenjing Hu ${ }^{d}$ Xiaoyun Fan ${ }^{d}$ Hua Liue Zhiming Zhu ${ }^{f}$ Dongfang Liu ${ }^{a}$ \\ Hongting Zheng ${ }^{g}$ Harvest F. Gu ${ }^{\text {h }}$ Rui Liu ${ }^{\mathrm{a}}$ Gangyi Yang ${ }^{\mathrm{a}}$
}

\begin{abstract}
aDepartment of Endocrinology, the Second Affiliated Hospital, Chongqing Medical University and Chongqing Clinical Research Center for Geriatrics, Chongqing, 'bCollege of Physical Education, Southwest University, Key Laboratory of Physical Evaluation and Sports Performance Monitoring of the State Administration of Sports, Chongqing, China, 'School of Biomedical Sciences, the University of Queensland, Brisbane, Australia, ${ }^{\mathrm{d} C h o n g q i n g}$ Prevention and Treatment Hospital for Occupational Diseases, Chongqing, China, 'Department of Pediatrics, University of Mississippi Medical Center, Jackson, USA, 'Department of Hypertension and Endocrinology, Daping Hospital, Third Military Medical University, Chongqing Institute of Hypertension, Chongqing, 9Department of Endocrinology, Xinqiao Hospital, Third Military Medical University, Chongqing, hSchool of Basic Medicine and Clinical Pharmacy, China Pharmaceutical University, Nanjing, China
\end{abstract}

\section{Key Words}

Alarin • Metabolic syndrome $\cdot$ Insulin resistance $\cdot$ Cytokine

\begin{abstract}
Background/Aims: Alarin has been reported to be related with increased food intake and body weight. The relationship of circulating Alarin with insulin resistance or metabolic syndrome (MetS), however, is unknown. This study aimed to investigate the physiological role of Alarin and its association with MetS in humans. Methods: Newly diagnosed MetS patients $(n=237)$ and age-matched healthy subjects $(n=192)$ were recruited for this study. Oral glucose tolerance test, treadmill exercise, lipid infusions and euglycemic-hyperinsulinemic clamp (EHCs) were performed. Circulating Alarin and TNF $\alpha$ levels were measured by ELISA. Results: Circulating Alarin levels were significantly higher in MetS patients compared with healthy subjects $(0.46 \pm 0.22$ vs. $0.41 \pm 0.14 \mu \mathrm{g} / \mathrm{L}, P<0.01)$. In all studied subjects, circulating Alarin levels were positively correlated with WC, blood pressure, FBG, triglyceride, HbA1c, HOMA-IR, $\mathrm{AUC}_{\text {glucose }}$ and TNFa $(P<0.05$ or $P<0.01)$. Multivariate logistic regression analyses revealed that circulating Alarin levels were correlated with MetS and insulin resistance. There was no significant change of circulating Alarin levels in the subjects with treadmill exercise for 45 min. In healthy individuals, however, glucose challenge, acute hyperglycemia and lipid infusions

$X$. Fang and T. Zhang contributed equally to this work.

Gangyi Yang and Rui Liu

Department of Endocrinology, the Second Affiliated Hospital, Chongqing Medical University and Chongqing Clinical Research Center for Geriatrics, 400010 Chongqing (China) Tel. +86-23-68485216, E-Mail gangyiyang@163.com; 317085605@qq.com
\end{abstract}


Fang et al.: Alarin and Metabolic Syndrome

resulted in increased circulating Alarin levels, while acute hyperinsulinaemia transiently decreased circulating Alarin levels. Conclusion: The present study provides the evidence that circulating Alarin levels are associated with MetS and insulin resistance.

(C) 2018 The Author(s)

Published by S. Karger AG, Basel

\section{Introduction}

Obesity and metabolic syndrome (MetS) are major health and socioeconomic problems that have reached epidemic proportions in the world [1]. Cumulative evidence indicates that obesity and MetS are two important risk factors for metabolic-related diseases, such as type 2 diabetes (T2D), coronary artery disease (CAD) and hypertension [2]. A number of studies have performed to address the connection between MetS and metabolic-related diseases in rodent models and humans. However, the mechanism underlying these links is unclear, partly because of the lack of appropriate animal models and the difficulty related human studies. Recently, a large number of adipokines and cytokines have been reported to be related to insulin resistance and MetS, such as betatrophin, zinc- $\alpha 2$-glycoprotein (ZAG) and myonectin [3-6]. Therefore, investigation of the relationship between new cytokines and MetS has an important clinical significance.

Alarin is a 25 amino acid cytokine, a member of the galanin peptide family isolated from the gangliocytes of human neuroblastic tumors, and is named after its $\mathrm{N}$-terminal alanine and its $\mathrm{C}$-terminal serine originating as a splice variant of galanin-like peptide (GALP) mRNA [7]. Alarin was initially found in ganglionic cells, and later on it has been found around the blood vessels with vasoactive actions for regulating ocular blood flow [8-10]. Subsequently, Alarin expression was found in the arcuate nucleus (ARC), a central nucleus related energy homeostasis in hypothalamus of rats [11]. Alarin peptide sequence is $92 \%$ similarity between mice and rats, and $96 \%$ between humans and monkeys [7]. In the animal study, Alarin has been reported to promote the secretion of luteinizing hormone (LH) [11] and has shown antidepressant-like and antimicrobial roles [12, 13]. In addition, peripheral and intraventricular (ICV) treatment of Alarin increased body weight and food intake, and promoted glucose uptake in muscle tissues mediated by insulin in rats [14-16]. Recently, Guo et al. also demonstrated that central treatment of Alarin increased adiponectin release, 2-(N-7-nitrobenz-2-oxa-1, 3-diazol-4-yl) amino-2-deoxyglucose uptake, glucose transporter 4 (Glut4) mRNA expression and translocation on cell membrane. During the hyperinsulinemic-euglycemic clamp (EHC), glucose infusion rates (GIR) in ICV Alarin-treated rats were increased, whereas blood glucose level and retinol-binding protein 4 (RBP4) were reduced in diabetic rats [17]. Therefore, Alarin has been considered as a cytokine related insulin resistance and energy homeostasis. However, so far, no study has demonstrated the relationship between circulating Alarin and MetS in humans.

In the current study, we investigated the possible role of circulating Alarin in humans and conducted the cross-sectional and interventional studies to evaluate whether Alarin is correlated with insulin resistance and MetS.

\section{Materials and Methods}

Cross-sectional study

This study was performed from Oct. 2015 to Oct. 2017. A total of 429 subjects (including 237 MetS and 192 healthy controls) were consecutively recruited to the study. MetS was diagnosed according to Executive Summary of The Third Report of The National Cholesterol Education Program (NCEP) Expert Panel on Detection, Evaluation, And Treatment of High Blood Cholesterol in Adults (Adult Treatment Panel III) criteria [18]. Patients with newly diagnosed MetS had not been treated with any medicines including lipid-lowering and hypoglycemic agents, as well as diet control or exercise. Excluded criteria included type 1 diabetic (T1D), liver cirrhosis, hepatic and renal failure, congestive heart failure, or other diseases. One hundred and ninety-two age-matched normal individuals without any major diseases were recruited from an unselected population of routine medical check-up, the community or schools as the controls. These 


\section{Cellular Physiology Cell Physiol Biochem 2018;51:2041-2051 and Biochemistry Published \begin{tabular}{l|l} 
DOI: 10.1159/000495823 & $\begin{array}{l}\text { () } 2018 \text { The Author(s). Published by S. Karger AG, Basel } \\
\text { www.karger.com/cpb }\end{array}$
\end{tabular} \\ Fang et al.: Alarin and Metabolic Syndrome}

subjects had no family history of T2D and hypertension. Healthy individuals had no taking any medications. A written voluntary consent was obtained from all subjects before their participation. The study complied with the Declaration of Helsinki and was approved by the Human Research Ethics Committee of Chongqing Medical University.

\section{Euglycemic-hyperinsulinemic clamp (EHC)}

EHCs were performed in thirty healthy individuals [ 15 men and 15 women; age $24 \pm 4$ yr; body mass index (BMI) $22.8 \pm 1.4 \mathrm{~kg} / \mathrm{m}^{2}$ ] as previously reported [3]. Briefly, after an overnight fast of $12-14 \mathrm{~h}$, two catheters were inserted into antecubital veins of the right and left arm for blood withdrawal or glucose and insulin infusions, respectively. Insulin ( $1 \mathrm{mU} / \mathrm{kg} / \mathrm{min}$, Humulin Regular; EliLilly) was infused for $2 \mathrm{~h}$, and $25 \%$ glucose was infused at variable rates that were adjusted to keep blood glucose levels at the baseline. During the EHCs, blood glucose was examined every $10 \mathrm{~min}$ to guide the glucose infusion. Glucose disposal rate (GRD) was defined as the glucose infusion rate (GIR) at the stable state of the EHC and was related to body weight (M-value). Blood samples were obtained at indicated times (0, 80, 100, 110 and $120 \mathrm{~min}$ ) [19].

\section{Lipid infusion study}

Twenty-two sex- and age-matched healthy subjects (11 men, 11 women) were assigned to receive a $20 \%$ Intralipid/heparin $(0.4 \mathrm{U} / \mathrm{kg} / \mathrm{min}$, Pharmacia and Upjohn, Milan, Italy) or a 0.9\% saline/heparin infusion (1.5 ml/minute) for 300 minutes. 3-hours after the start of the lipid or saline/heparin infusion, a 2-hour EHC was performed as described above. Blood samples for further analysis were drawn before and at indicated timed intervals during lipid infusion and the EHCs.

\section{Exercise testing procedures}

A subgroup of 12 healthy volunteers ( 6 men and 6 women, aged 20-25; BMI: $21.0 \pm 1.2 \mathrm{~kg} / \mathrm{m}^{2}$ ) was assigned to a 45-min bout of treadmill exercise. After an overnight fast for 10-12 h, individuals were asked to arrive at the laboratory between 8:00 and 9:00 on the morning of the treadmill exercise study. All study subjects performed a 45-min bout of treadmill exercise using ParvoMedics Metabolic Measurement System (ParvoMedics, Sandy, Utah, USA) at $60 \%$ of $\mathrm{VO}_{2}$ max. Blood samples were obtained from a forearm intravenous catheter at 4 time points including baseline, $45 \mathrm{~min}$ following treadmill exercise, 60 and 120 min at rest after the treadmill exercise.

\section{The daily secretion measurement}

Twelve healthy volunteers ( 6 men and 6 women) aged 20-30 were arranged to the ward at 0740 and were given with a standardized breakfast. During at indicated times $(0800,1000,1200,1600,2000,2400$, and 0400), blood samples were drawn from the forearm vein over a period of $24 \mathrm{~h}$ to observe the daily varieties of Alarin concentration. Blood samples were immediately centrifuged, separated and stored at $-80^{\circ} \mathrm{C}$ for measuring Alarin concentration.

\section{Measurements of adipokines}

Serum Alarin levels were measured with a commercial ELISA Kit following the manufacturer's protocol (Phoenix Pharmaceuticals, Inc., Belmont, CA, USA). The kit has a sensitivity of $0.08 \mathrm{ng} / \mathrm{ml}$ and a linear range of $0.08-0.78 \mathrm{ng} / \mathrm{ml}$. Intra-assay and inter-assay variations were $<10 \%$ and $<15 \%$, respectively. The ELISA kit had been validated by the dealer, showing high sensitivity and excellent specificity for detection of human Alarin, but no significant cross-reactivity with other members of the galanin peptide family. Serum tumor necrosis factor- $\alpha$ (TNF $\alpha$ ) concentrations were measured by ELISA Kit (4A Biotech Co. Ltd, Beijing, China). The linear range of the ELISA assay was 4.3-1000 ng/L. The intra- and inter-assay coefficients (CV) were both $<10 \%$.

\section{Anthropometric and biochemical evaluation}

Anthropometric and body composition were measured by a trained dietician in all subjects after an overnight. BMI and the waist-to-hip ratio (WHR) were calculated as reported previously [20]. The homeostasis model assessment of insulin resistance (HOMA-IR) and the area under the curve for glucose $\left(\mathrm{AUC}_{\text {glucose }}\right)$ and insulin $\left(\mathrm{AUC}_{\text {Insulin }}\right)$ were calculated as reported previously [21]. Blood glucose, HbA1c, Free fatty acids (FFAs), total cholesterol (TC), high-density lipoprotein cholesterol (HDL-C), low-density lipoprotein cholesterol (LDL-C), and triglyceride (TG) were determined as published previously [20].

\section{Statistical Analysis}

All analyses were performed using SPSS version 19.0 (SPSS, Chicago, IL, USA). Normal distribution of the data was tested using Kolmogorox-Smirnov test. The variables of non-normal distribution were skewed and logarithmically transformed to obtain a normal distribution. Continuous variables were presented as 
mean and standard deviation (SD) or median and interquartile range (IQR). Comparisons between groups were performed using ANOVA, an unpaired $t$-test or a paired $t$-test. Simple and multiple linear regression analyses were used to assess the association between Alarin and the other biomarkers. The utility of circulating Alarin to predict MetS and insulin resistance was evaluated by receiver operating characteristic (ROC) curve analysis. Sample size was calculated using the following equation: $\mathrm{N}=\left[\mathrm{Z}_{\alpha / 2} \sigma / \varepsilon \mu\right]^{2}(\sigma$, standard deviation; $\mu$, mean; $\mathrm{Z}_{\alpha / 2}=1.96, \alpha=0.05, \varepsilon=4 \%$ ). In all statistical tests, $P$ values of $<0.05$ were considered significant.

\section{Results}

\section{Circadian rhythm of circulating Alarin in healthy controls}

Twelve normal controls were given a standardized breakfast (0730), and blood samples were collected from 0800 on day 1 to 0400 on day 2 to observe whether serum Alarin levels are regulated by circadian factors. Data showed that serum Alarin decreased to nadir at 12:00 $\mathrm{h}$, and then started to rise at 16:00 h, peaked at 24:00 h. Alarin started to decrease from 4:00 h to 8:00 h (Fig. 1A). Therefore, these data showed that the circadian rhythm of circulating Alarin was smooth and there is no big fluctuation.

\section{Circulating Alarin levels and its association with other parameters}

Anthropometric and biochemical parameters of the 429 subjects were shown in Table 1. MetS patients had higher BMI, WHR, blood pressure (BP), TG, FFA, fasting blood glucose (FBG), 2-h post-glucose load blood glucose (2h-BG), HbA1c, fasting insulin (FIns), 2-h insulin after glucose overload (2h-Ins), HOMA-IR, $\mathrm{AUC}_{\text {glucose }}$ and TNF- $\alpha$, and lower HDL-C than the

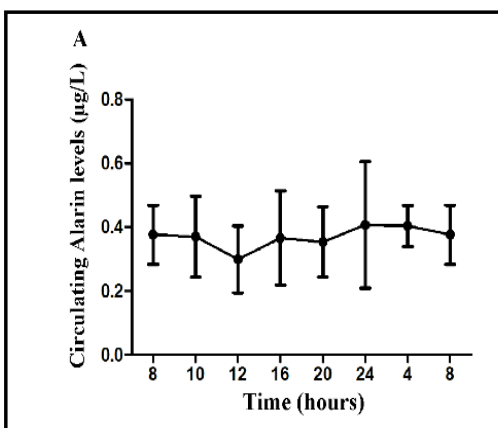

D
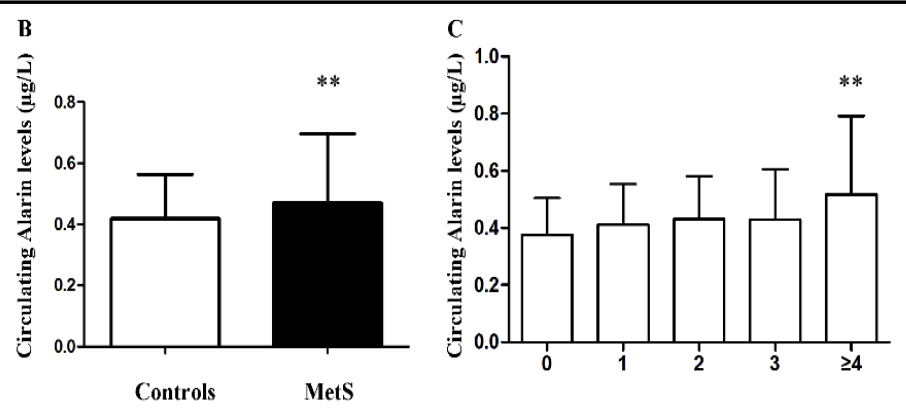

MetS

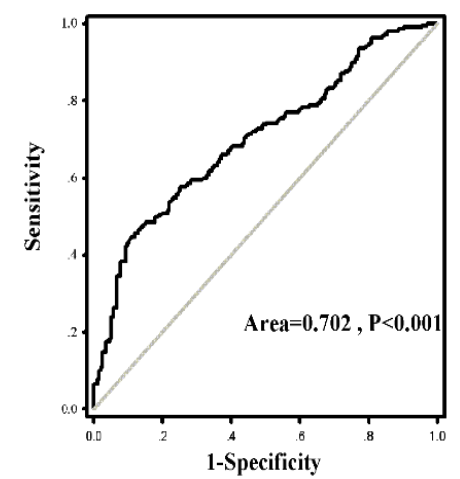

E

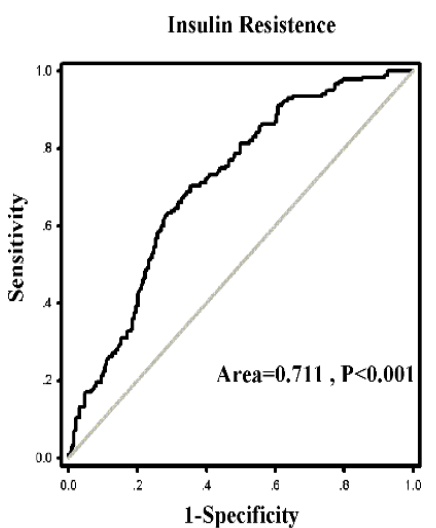

Fig. 1. Concentration of circulating Alarin in study population. A. Circadian of circulating Alarin variations in healthy subjects. B. Circulating Alarin levels according to MetS and controls. C. Circulating concentrations of Alarin elevated with increasing number of components of the MetS. Values were given as means \pm SD, ${ }^{*} \mathrm{P}<0.05$, ${ }^{* *} \mathrm{P}<0.01$ compared with controls or 0 component. D-E. ROC curve analyses were performed for the prediction of circulating Alarin for MetS. D. and insulin resistance E. 
controls. Importantly, circulating Alarin concentrations were higher in MetS subjects than in controls $(P<0.01$, Fig. 1B). The difference remained significant after adjustment for sex and age (Table 1). Next, the association of Alarin with other variables was analyzed by partial correlations. In all study population, circulating Alarin closely correlated with waist circumference (WC) $(P<0.05)$, BP $(P<0.05)$, parameters of blood glucose (FBG and HbA1c, both $P<0.05$ ), insulin resistance indices (increased HOMA-IR and AUC $_{\text {glucose }} P<0.05$ or $P<0.01$ ), an adverse lipid profile (increased TG, $P<0.01$ ) and inflammatory markers (TNF- $\alpha, P<0.05$; Table 2 ). These associations remained statistically significant even after adjusting for age and BMI, except for TNF- $\alpha$.

In multiple stepwise regression analyses, we found that serum Alarin levels were independently associated with the two important components of MetS, SBP $(\beta=0.001, P<$ $0.05)$ and FBG $(\beta=0.028, P<$ $0.01)$, and $\mathrm{AUC}_{\text {glucose }}(\beta=0.011$, $P<0.001)$. Multivariate logistic regression analysis found that serum Alarin concentrations were markedly related MetS and insulin resistance, even after adjustment for anthropometric parameters, age, gender and BP (Table 3).

To further investigate the association between Alarin and the occurrence of MetS, we stratified the concentrations of serum Alarin by the number of MetS components. The results showed that serum Alarin levels were significantly elevated when the number of MetS components was equal to 4 or more (Fig. 1C). Individuals with 4 or more MetS components had Alarin levels (mean \pm SD) of $0.51 \pm 0.26 \mu \mathrm{g} / \mathrm{L}$, or higher. The ORs for MetS components associated with circulating Alarin were showed in Table 4. Of the five MetS components, hypertriglyceridemia, hyperglycemia and central obesity seem to have the positive associations with circulating Alarin levels in women, while hyperglycemia and HOMA-IR in men.

Finally, we performed the ROC curve analyses. The results showed that the best cut-off value for Alarin to predict MetS was $0.47 \mu \mathrm{g} / \mathrm{L}$ (sensitivity $65 \%$, specificity $89 \%$, and AUC 0.702 ; Fig. 1D), and to predict insulin resistance was $0.41 \mu \mathrm{g} / \mathrm{L}$ (sensitivity $65 \%$, specificity $71 \%$, and AUC 0.711, Fig. 1E).

Table 1. Main clinical features and circulating Alarin levels in study population MetS, metabolic syndrome; BMI, body mass index; WHR, waist-to-hip ratio; FAT\%, visceral fat\%; SBP, systolic blood pressure; DBP, diastolic blood pressure; TG, triglyceride; TC, total cholesterol; HDL-C, high-density lipoprotein cholesterol; LDL-C, low-density lipoprotein cholesterol; FFA, free fatty acid; FBG, fasting blood glucose; $2 \mathrm{~h}-\mathrm{BG}, 2 \mathrm{~h}$ post-glucose load blood glucose; FIns, fasting insulin; $2 \mathrm{~h}$-Ins, $2 \mathrm{~h}$ serum insulin after glucose overload; HOMA-IR, HOMA-insulin resistance index; $\mathrm{AUC}_{\text {insulin }}$, the area under the curve for insulin; $\mathrm{AUC}_{\text {glucose }}$ the area under the curve for glucose; TNF- $\alpha$, tumor necrosis factor- $\alpha$. Data are present as mean (standard deviation) or median (interquartile range). ${ }^{1}$ Mean and standard error by general linear model with adjustment of age and gender

\begin{tabular}{|c|c|c|c|}
\hline Group & Controls (N=192) & MetS (N=237) & $\mathrm{P}$ value \\
\hline Age (yr) & $54.2(9.6)$ & $55.4(9.9)$ & 0.239 \\
\hline BMI $\left(\mathrm{kg} / \mathrm{m}^{2}\right)$ & $22.8(2.8)$ & $25.1(3.1)$ & $\begin{array}{c}< \\
0.001\end{array}$ \\
\hline WHR & $0.87(0.08)$ & $0.92(0.05)$ & $\begin{array}{c}< \\
0.001\end{array}$ \\
\hline TNF- $\alpha$ & $7.58(5.40-15.71)$ & $8.64(5.38-16.95)$ & $<0.05$ \\
\hline SBP (mmHg) & $118.0(15.3)$ & $132.3(16.7)$ & $\begin{array}{c}< \\
0.001\end{array}$ \\
\hline DBP (mmHg) & $74.8(10.2)$ & $81.5(11.0)$ & $\begin{array}{c}< \\
0.001\end{array}$ \\
\hline $\mathrm{TG}(\mathrm{mmol} / \mathrm{L})$ & $1.23(0.85-1.40)$ & $1.89(1.49-2.75)$ & $\begin{array}{c}< \\
0.001\end{array}$ \\
\hline $\mathrm{TC}(\mathrm{mmol} / \mathrm{L})$ & $5.09(0.94)$ & $5.08(1.04)$ & 0.901 \\
\hline HDL-C (mmol/L) & $1.42(0.28)$ & $1.17(0.31)$ & $\begin{array}{c}< \\
0.001\end{array}$ \\
\hline $\mathrm{LDL}-\mathrm{C}(\mathrm{mmol} / \mathrm{L})$ & $3.02(0.79)$ & $2.95(0.90)$ & 0.439 \\
\hline FFA $(\mu \mathrm{mol} / \mathrm{L})$ & $0.49(0.39-0.69)$ & $0.59(0.43-0.89)$ & $\begin{array}{c}< \\
0.001\end{array}$ \\
\hline $\mathrm{FBG}(\mathrm{mmol} / \mathrm{L})$ & $6.23(2.76)$ & $7.73(3.27)$ & $\begin{array}{c}< \\
0.001\end{array}$ \\
\hline 2h-BG (mmol/L) & $7.41(6.04-10.67)$ & $11.86(8.59-19.10)$ & $\begin{array}{c}< \\
0.001\end{array}$ \\
\hline FIns (mU/L) & $9.06(5.09)$ & $13.1(7.50)$ & $\begin{array}{c}< \\
0.001\end{array}$ \\
\hline 2h-Ins (mU/L) & $46.82(30.92-71.98)$ & $69.47(37.20-117.10)$ & $\begin{array}{c}< \\
0.001\end{array}$ \\
\hline AUCinsulin & $100.4(67.21-146.42)$ & $119.9(68.07-165.14)$ & 0.229 \\
\hline AUC glucose & $17.0(13.60-22.03)$ & $24.9(18.92-32.66)$ & $\begin{array}{c}< \\
0.001\end{array}$ \\
\hline HbA1c (\%) & $6.19(1.66)$ & 7.07 (1.97) & $\begin{array}{c}< \\
0.001\end{array}$ \\
\hline HOMA-IR & $1.97(1.33-2.93)$ & $3.62(2.60-5.70)$ & $\begin{array}{c}< \\
0.001\end{array}$ \\
\hline Alarin $(\mu \mathrm{g} / \mathrm{L})$ & $0.41(0.14)$ & $0.46(0.22)$ & $\begin{array}{c}< \\
0.001\end{array}$ \\
\hline Alarin $(\mu \mathrm{g} / \mathrm{L})^{1}$ & $0.42(0.01)$ & $0.47(0.01)$ & $\begin{array}{c}< \\
0.001\end{array}$ \\
\hline
\end{tabular}

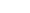


The effects of acute exercise and glucose challenge on circulating Alarin levels in healthy subjects

To explore which factors regulate serum Alarin concentrations in vivo, we examined the effects of a single exercise and glucose challenge on Alarin in healthy controls. For acute exercise study, a 45 -min bout of treadmill exercise was performed in 12 subjects at $60 \%$ of $\mathrm{VO}_{2} \max$, and then rested for $120 \mathrm{~min}$. After a 45-min exercise, there was no obvious change in the circulating levels of Alarin as compared to pre-exercise $(0.36 \pm 0.09$ vs. $0.35 \pm$ $0.10 \mu \mathrm{g} / \mathrm{L}$, Fig. 2A). During the 2-h OGTT, in response to oral glucose challenge-induced hyperglycemia and hyperinsulinemia, Alarin concentrations gradually increased from $0.36 \pm 0.10 \mu \mathrm{g} / \mathrm{L}$ at $0 \mathrm{~min}$, and to $0.43 \pm 0.09 \mu \mathrm{g} / \mathrm{L}$ at $30 \mathrm{~min}$, and then to $0.42 \pm 0.10 \mu \mathrm{g} / \mathrm{L}$ at $60 \mathrm{~min}$, and finally to $0.43 \pm 0.11 \mu \mathrm{g} / \mathrm{L}$ at 120 min (vs. 0 min $P<0.05$, Fig. 2B).

\section{The effects of hyperinsulinemia} and lipid-induced insulin resistance on Alarin levels in normal subjects

To examine whether serum Alarin is impacted by euglycemichyperinsulinemic state, we performed EHCs in 30 normal controls. During the steady-state of clamp, blood glucose was maintained at $\sim 5 \mathrm{mmol} / \mathrm{L}$ and insulin was elevated from $44.5 \pm$ 10.2 to $315.3 \pm 48.2 \mathrm{pmol} / \mathrm{L}$. In response to hyperinsulinemia, the GIR needed to maintain euglycemia (expressed as M-values) elevated from 0 to $10.4 \pm 2.8 \mathrm{mg} / \mathrm{kg} / \mathrm{min}$ in these subjects. However, in the euglycemic-hyperinsulinemic state, serum Alarin levels showed a transient decrease, and then returning to pre-EHC levels (from $0.30 \pm 0.05 \mu \mathrm{g} / \mathrm{L}$ at $0 \mathrm{~min}$, to $0.28 \pm 0.05 \mu \mathrm{g} / \mathrm{L}$ at $80 \mathrm{~min}$, and to $0.26 \pm 0.06 \mu \mathrm{g} / \mathrm{L}$ at $100 \mathrm{~min}, v s .0 \mathrm{~min} P<0.05$, and then increasing to $0.29 \pm$ $0.07 \mu \mathrm{g} / \mathrm{L}$ at $110 \mathrm{~min}$, and finally to $0.29 \pm 0.05 \mu \mathrm{g} / \mathrm{L}$ at $120 \mathrm{~min}$ ) (Fig. 2C). Fig. 2D showed the average level of circulating Alarin during the EHC, suggesting that Alarin levels were decreased by insulin stimulated.

To investigate the effect of lipid-induced insulin resistance on circulating Alarin in vivo, we examined the alterations of serum Alarin level before and after lipid infusion combined with EHCs in 22 normal individuals. As expected, GIR was significantly decreased in lipid

Table 2. Partial correlations analysis of variables associated with circulating Alarin levels in study population MetS, metabolic syndrome; BMI, body mass index; WHR, waist-tohip ratio; FAT \%, visceral fat \%; SBP, systolic blood pressure; DBP, diastolic blood pressure; TG, triglyceride; TC, total cholesterol; HDL-C, high-density lipoprotein cholesterol; LDL-C, low-density lipoprotein cholesterol; FFA, free fatty acid; FBG, fasting blood glucose; $2 \mathrm{~h}-\mathrm{BG}, 2 \mathrm{~h}$ post-glucose load blood glucose; FIns, fasting insulin; $2 \mathrm{~h}$-Ins, $2 \mathrm{~h}$ serum insulin after glucose overload; HOMA-IR, HOMA-insulin resistance index; $\mathrm{AUC}_{\text {insulin }}$, the area under the curve for insulin; $\mathrm{AUC}_{\text {glucose' }}$ the area under the curve for glucose; TNF- $\alpha$, tumor necrosis factor- $\alpha$. Data are present as mean (standard deviation) or median (interquartile range)

\begin{tabular}{lcccccc}
\hline Item & \multicolumn{2}{c}{ Alarin } & \multicolumn{2}{c}{ Alarin } & \multicolumn{2}{c}{ Alarin } \\
& $\mathrm{R}$ & P value & $\mathrm{R}$ & $\mathrm{R}$ & P value & \multicolumn{2}{c}{ R } & P value \\
\hline Age & 0.003 & 0.980 & & & & \\
BMI & 0.124 & 0.216 & 0.125 & 0.213 & & \\
WC & 0.221 & $<0.05$ & 0.222 & $<0.05$ & 0.200 & $<0.05$ \\
WHR & 0.081 & 0.417 & 0.082 & 0.417 & 0.054 & 0.593 \\
SBP & 0.222 & $<0.05$ & 0.236 & $<0.05$ & 0.208 & $<0.05$ \\
DBP & 0.199 & $<0.05$ & 0.199 & $<0.05$ & 0.177 & 0.078 \\
FBG & 0.249 & $<0.05$ & 0.249 & $<0.05$ & 0.249 & $<0.05$ \\
FIns & 0.049 & 0.627 & 0.049 & 0.624 & -0.004 & 0.971 \\
HOMA-IR & 0.143 & $<0.05$ & 0.145 & $<0.05$ & 0.097 & $<0.05$ \\
HbA1c & 0.241 & $<0.05$ & 0.243 & $<0.05$ & 0.238 & $<0.05$ \\
TG & 0.271 & $<0.01$ & 0.272 & $<0.01$ & 0.250 & $<0.05$ \\
TC & 0.083 & 0.406 & 0.083 & 0.408 & 0.070 & 0.491 \\
HDL-C & -0.150 & 0.121 & -0.156 & 0.120 & -0.129 & 0.199 \\
LDL-C & 0.040 & 0.689 & 0.040 & 0.691 & 0.026 & 0.800 \\
FFA & 0.032 & 0.746 & 0.033 & 0.746 & 0.025 & 0.804 \\
AUCinsulin & 0.086 & 0.390 & 0.087 & 0.385 & 0.060 & 0.555 \\
AUC glucose & 0.298 & $<0.01$ & 0.298 & $<0.01$ & 0.284 & $<0.01$ \\
TNF- $\alpha$ & 0.133 & $<0.05$ & 0.133 & $<0.05$ & 0.124 & 0.068 \\
\hline
\end{tabular}

Table 3. Association of circulating Alarin with MetS and insulin resistance in fully adjusted models $\mathrm{CI}$, confidence interval; OR, odds ratio; MetS, metabolic syndrome; IR, HOMA-IR. Results of multivariate logistic regression analysis are presented as the odds ratio of being in MetS and IR status increase in serum Alarin

\begin{tabular}{lcccccc}
\hline \multirow{2}{*}{ Model adjust } & \multicolumn{3}{c}{ Mets } & \multicolumn{3}{c}{ Insulin resistance } \\
& OR & $95 \%$ CI & P value & OR & $95 \%$ CI & P value \\
\hline Age & 4.27 & $1.40-12.99$ & $<0.01$ & 4.01 & $1.29-12.44$ & $<0.01$ \\
Age, Sex & 4.47 & $1.45-13.78$ & $<0.01$ & 3.83 & $1.23-11.89$ & $<0.01$ \\
Age, Sex, BP & 4.19 & $1.23-14.26$ & $<0.05$ & 3.89 & $1.21-12.46$ & $<0.01$ \\
Age, Sex, BP, BMI & 4.22 & $1.17-15.17$ & $<0.05$ & 3.74 & $1.12-12.47$ & $<0.01$ \\
Age, Sex, BP, BMI, WHR & 4.44 & $1.21-16.34$ & $<0.05$ & 3.79 & $1.13-12.68$ & $<0.01$ \\
Age, Sex, BP, BMI, WHR, IR & 2.50 & $0.68-9.23$ & 0.169 & & & \\
\hline
\end{tabular}
KARGER 
Fang et al.: Alarin and Metabolic Syndrome

Table 4. Odds ratios and $95 \%$ confidence interval (CI) of circulating Alarin for individual components of MetS in male and female subjects Data are present as median and interquartile range

\begin{tabular}{|c|c|c|c|c|c|c|}
\hline \multirow{2}{*}{$\begin{array}{l}\text { Individual } \\
\text { components } \\
\text { of MetS }\end{array}$} & \multicolumn{2}{|c|}{ Men $(\mathrm{N}=165)$} & \multicolumn{2}{|c|}{ Women $(\mathrm{N}=264)$} & \multicolumn{2}{|c|}{ Total $(\mathrm{N}=429)$} \\
\hline & OR $(95 \% \mathrm{CI})$ & $P$ value & OR $(95 \% \mathrm{CI})$ & $P$ value & OR $(95 \% \mathrm{CI})$ & $P$ value \\
\hline All components & $2.01(0.39-10.37)$ & 0.40 & 9.07 (1.81-45.48) & $\begin{array}{c}< \\
0.001\end{array}$ & $4.35(1.43-13.24)$ & $\begin{array}{c}< \\
0.01\end{array}$ \\
\hline $\begin{array}{l}\text { Hypertension } \\
\text { High HDL-C }\end{array}$ & $\begin{array}{l}1.66(0.32-8.61) \\
0.46(0.08-2.60)\end{array}$ & $\begin{array}{l}0.54 \\
0.38\end{array}$ & $\begin{array}{c}1.26(0.34-4.57) \\
2.50(0.62-10.08)\end{array}$ & $\begin{array}{l}0.72 \\
0.19\end{array}$ & $\begin{array}{l}1.51(0.55-4.15) \\
1.09(0.40-2.96)\end{array}$ & $\begin{array}{l}0.41 \\
0.86\end{array}$ \\
\hline Hypertriglyceridemia & $1.59(0.31-8.17)$ & 0.57 & $5.61(1.38-22.75)$ & $<0.01$ & $3.63(1.27-10.38)$ & $\begin{array}{c}< \\
0.01\end{array}$ \\
\hline Hyperglycemia & $\begin{array}{c}351.52(13.53- \\
9126.95)\end{array}$ & $\begin{array}{c}< \\
0.001\end{array}$ & $10.44(1.87-58.16)$ & $\begin{array}{c}< \\
0.001\end{array}$ & $\begin{array}{c}33.90(7.16- \\
160.51)\end{array}$ & $\begin{array}{c}< \\
0.01\end{array}$ \\
\hline Central obesity & $1.27(0.25-6.39)$ & 0.76 & $10.15(1.48-69.20)$ & $<0.01$ & $2.35(0.78-7.06)$ & 0.12 \\
\hline HOMA-IR & $8.61(1.19-62.21)$ & $<0.05$ & $\begin{array}{c}2.414(0.615- \\
9.474)\end{array}$ & 0.414 & $3.99(1.29-12.340)$ & $\begin{array}{c}< \\
0.05\end{array}$ \\
\hline
\end{tabular}

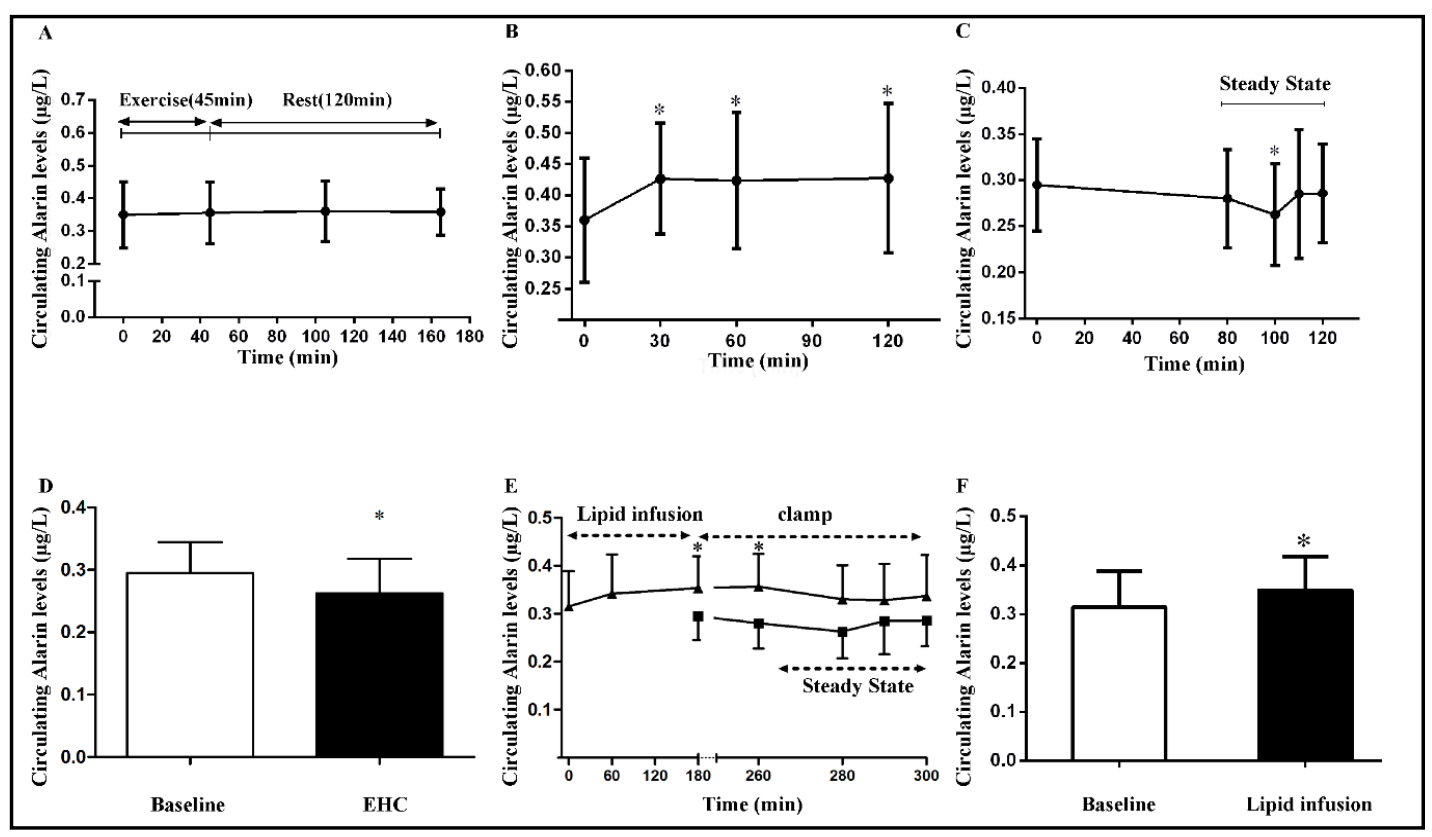

Fig. 2. Circulating Alarin levels in interventional studies. A. Circulating Alarin in healthy individuals in response to acute exercise. B. Circulating Alarin concentrations in healthy subjects during an OGTT with 75 g glucose. C. Time course of circulating Alarin changes in healthy subjects during EHC. D. Circulating Alarin levels in the basal and insulin- stimulated state. E. Time course of circulating Alarin changes in healthy subjects during lipid infusion combined with EHC. F. The changes of circulating Alarin levels at baseline and during lipid infusion in healthy subjects. Data are means \pm SD. $*$ P $<0.05$ vs. baseline.

infused individuals than in controls $(4.53 \pm 1.86$ vs. $9.58 \pm 2.40 \mathrm{mg} / \mathrm{kg} / \mathrm{min}, P<0.01)$. Importantly, serum Alarin levels were significantly increased from 0 to $180 \mathrm{~min}$ during lipid infusion [from $0.31 \pm 0.07$ to $0.35 \pm 0.07 \mu \mathrm{g} / \mathrm{L} ; P<0.05$; Fig. 2E]. The average Alarin levels during intralipid infusion were shown in Fig. $2 \mathrm{~F}(P<0.05)$. During the last 3 hour of lipid infusion and 2 hour of EHC, hyperinsulinemia led to a transient decrease of circulating Alarin levels (from $0.35 \pm 0.07$ at $260 \mathrm{~min}$ to $0.33 \pm 0.07 \mu \mathrm{g} / \mathrm{L}$ at $280 \mathrm{~min}$, Fig. $2 \mathrm{E}$ ).

\section{Discussion}

In animal studies, the beneficial effects of Alarin on ameliorating insulin resistance, decreasing insulin levels and blood glucose have been revealed in the vivo and vitro [16,17]. 
However, the relationship between circulating Alarin and metabolic diseases has not been reported in humans. To better demonstrate the association of the circulating Alarin levels with MetS and to avoid the interference of clinical confounding factors, newly diagnosed MetS individuals were recruited in the current study. Therefore, the effects of disease duration, diet and medicine were excluded. Here, we demonstrated that circulating Alarin levels were markedly increased in MetS individuals relative to healthy controls and circulating Alarin is independently associated with MetS and insulin resistance. These results reveal a relationship between circulating Alarin and MetS and suggest that a high level of Alarin might be beneficial to MetS in humans. However, the mechanisms underlying increased Alarin levels in MetS subjects remain elusive.

In a previous study, we reported that circulating Sfrp5, an adipokine, was significantly lower in MetS individuals and it may predict for MetS [22]. In the current study, we observed that circulating Alarin levels were significantly higher in MetS individuals. Therefore, when an individual has higher circulating Alarin levels and low Sfrp5 levels, it is more likely to be diagnosed as MetS.

Alarin has recently been found to be relative to the regulation of energy balance [23]. We speculate that the elevation of circulating Alarin in MetS individuals might be a compensatory up-regulation in vivo for counteracting the metabolic stress produced by obesity, hyperglycemia, or hyperlipemia. Furthermore, it is also possible that adiposity or metabolic disorder may cause the resistance of Alarin actions, like insulin or leptin resistances, leading to the increase of Alarin secretion and release.

Our cross-sectional study has showed an independent association of the circulating Alarin with other metabolic risk factors, including abdominal obesity, insulin resistance, dyslipidemia, hyperglycemia and hypertension. These findings indicate that Alarin might have a regulatory role in glucose and lipid metabolism in vivo, consistent with the results of two animal studies $[16,17]$. Therefore, the elevation of circulating Alarin levels in MetS subjects might be beneficial to the improvement of metabolism in vivo.

To investigate the possible association of the five components of MetS and circulating Alarin, we performed logistic regression analysis. Hypertriglyceridemia, hyperglycemia and central obesity seemed to have the positive associations with Alarin in women. In keeping with this finding, animal study reported that Alarin was associated with body weight [15], glucose uptake and blood glucose levels [16]. Therefore, the results suggest an awareness of the need to screen population with MetS for the presence of high Alarin levels.

In our ROC curve analysis, the results show that circulating Alarin may predict both MetS and insulin resistance in our study population. In one aspect, the range of AUC (0.60.7 ) was considered to be mild-to-moderate significance, which may be due to the influence of the sample size and a non-normal distribution of Alarin levels in the studied population. In another aspect, the circulating Alarin may not be a good marker for predicting MetS and insulin resistance. Therefore, further extensive study is necessary for making a solid conclusion.

An important finding of this study is a significant increase of serum Alarin levels after an oral glucose challenge in normal subjects. This pattern is similar to insulin and glucose during OGTT, suggesting a reciprocal regulation among these factors in response to metabolic changes. The increase of circulating Alarin during OGTT may be due to increased glucose and/or insulin. To rule out possible effects of elevate insulin levels produced by oral glucose challenge on circulating Alarin, we performed EHC (a euglycemic-hyperinsulinemic state) on 30 normal subjects. During the steady-state of EHC, circulating Alarin levels showed a transient change. It decreased at $100 \mathrm{~min}$ of EHC, followed by a gradual return to baseline. These results lead us to speculate that short-term hyperinsulinemia may have an inhibitory effect on Alarin secretion and/or release. Therefore, the elevated Alarin concentrations during oral glucose challenge are likely due to the increased glucose levels rather than insulin, as increased blood glucose may be sufficient to eliminate the effect of insulin on circulating Alarin. 
Insulin resistance is an important feature of some metabolic disorders like obesity, dyslipidemias, T2D, hypertension and MetS, all of which are risk factors for CAD [24]. It is well documented that lipid infusion induces insulin resistance in humans via inhibition of glucose transport activity $[3,25]$. To further investigate whether circulating Alarin is related to insulin resistance in humans, intralipid infusion was performed in normal individuals. We observed that intralipid infusion led to an increase in serum Alarin concentrations and then a decrease during EHC. Therefore, circulating Alarin showed a biphasic change during lipid infusion with EHC. These findings revealed that acute elevation of FFA by lipid infusion contributed to the elevated Alarin levels, and subsequent hyperinsulinaemia during EHC led to a reduction in Alarin levels. These data demonstrate that FFA regulate Alarin release and support a role for Alarin in FFA-induced insulin resistance. Insulin resistance induced by FFAs is principally due to substrate competition and intracellular biochemical pathways including glucose phosphorylation and regulation of pyruvate dehydrogenase complex. Thus, it is possible to consider that Alarin may exert a role in insulin resistance during T2D independent of hyperinsulinaemia.

It is generally accepted that the metabolic regulation in obesity, T2D and MetS patients is influenced by their diet and physical activity, and physical activity protects against several types of disease including CAD, T2D and MetS [26, 27]. Recently, muscle tissues have been found to be an endocrine organ that releases cytokines, named myokines. Myokines have been shown to modulate several physiological pathways [28]. To explore whether Alarin secretion was increased by muscle tissue through physical activity, we investigated the effect of acute exercise on circulating Alarin. However, we observed that a 45-min bout of exercise in healthy individuals was not effective in modulating Alarin levels. This finding suggests that the release of Alarin may not be regulated by muscle tissues and this peptide may not be an exercise- responsive myokine. However, the effects of long-term exercise on circulating Alarin levels are unknown, and further studies are necessary.

There are some limitations in our research. First, the cross-sectional design of this study does not allow us to deduce a causal relationship between Alarin and MetS. Prospective studies are needed to clarify their precise interrelationship. Second, Alarin concentrations were not a pre-specified end point for recruited MetS subjects. Finally, our results could be influenced by some outliers due to the sample size. Despite these limitations, this study demonstrated clinically significant associations and differences between groups.

\section{Conclusion}

In summary, the present study provided evidence that circulating Alarin levels was significantly elevated in newly diagnosed MetS individuals and the circulating Alarin was impacted by oral glucose challenge, acute hyperinsulinemia and lipid infusion. Therefore, the data indicated that Alarin might be a cytokine related to nutrition and metabolism. Further large-scale prospective studies including different ethnic groups have been taken into our consideration.

\section{Abbreviations}

EHCs (euglycemic-hyperinsulinemic clamp); TNF- $\alpha$ (tumor necrosis factor- $\alpha$ ); (WC, waist circumference); FBG (fasting blood glucose); HOMA-IR (homeostasis model assessment of insulin resistance); $\mathrm{AUC}_{\text {glucose }}$ (the area under the curve for glucose). 


\section{Cellular Physiology Cell Physiol Biochem 2018;51:2041-2051 and Biochemistry Published ond $\begin{aligned} & \text { DOI: 10.1159/000495823 } \\ & \text { (c) } 2018 \text { The Author(s). Published by S. Karger AG, Basel } \\ & \text { www.karger.com/cpb }\end{aligned}$}

Fang et al:: Alarin and Metabolic Syndrome

\section{Acknowledgements}

This work was supported by research grants from the National Natural Science Foundation of China (81670755, 81601214 and 81300670), Natural Science Foundation Key Project of CQcstc (cstc2015jcyjA10084), Science and Technology Key Program of Health Bureau of Chongqing (2015ZDXM038, 2016MSXM083), and Science and technology program of Chongqing Nanan District Health Bureau (201619).

\section{Disclosure Statement}

The authors declare that there are no conflicts of interest.

\section{References}

1 Ng M, Fleming T, Robinson M, Thomson B, Graetz N, Margono C: Global, regional, and national prevalence of overweight and obesity in children and adults during 1980-2013: a systematic analysis for the Global Burden of Disease Study 2013. Lancet 2014;384:766-781.

2 Tremblay M, Brisson D, Gaudet D: Association between salivary pH and metabolic syndrome in women: a cross-sectional study. BMC Oral Health 2012;12:40.

- Luo Y, Li L, Xu X, Wu T, Yang M, Zhang C, Mou H, Zhou T, Jia Y, Cai C, Liu H, Yang G, Zhang X: Decreased circulating BMP-9 levels in patients with Type 2 diabetes is a signature of insulin resistance. Clin Sci (Lond) 2017;131:239-246.

4 Li K, Liao X, Wang K, Mi Q Zhang T, Jia Y, Xu X, Luo X, Zhang C, Liu H, Li L, Yang G: Myonectin Predicts the Development of Type 2 Diabetes. J Clin Endocrinol Metab 2018;103:139-147.

5 Lei L, Li K, Li L, Fang X, Zhou T, Zhang C, Luo Y, Liu H, Li X, Zheng H, Zhang L, Yang G, Gao L: Circulating zinc$\alpha 2$-glycoprotein levels are low in newly diagnosed patients with metabolic syndrome and correlate with adiponectin. Nutr Metab (Lond) 2017;14:53.

6 Abu-Farha M, Abubaker J, Al-Khairi I, Cherian P, Noronha F, Kavalakatt S, Khadir A, Behbehani K, Alarouj M, Bennakhi A, Elkum N: Circulating angiopoietin-like protein 8 (betatrophin) association with HsCRP and metabolic syndrome. Cardiovasc Diabetol 2016;15:25.

7 Santic R, Fenninger K, Graf K, Schneider R, Hauser-Kronberger C, Schilling FH, Kogner P, Ratschek M, Jones N, Sperl W, Kofler B: Gangliocytes in neuroblastic tumors express alarin, a novel peptide derived by differential splicing of the galanin-like peptide gene. J Mol Neurosci 2006;29:145-152.

8 Santic R, Schmidhuber SM, Lang R, Rauch I, Voglas E, Eberhard N, Bauer JW, Brain SD, Kofler B: Alarin is a vasoactive peptide. Proc Natl Acad Sci USA 2007;104:10217-10222.

-9 Eberhard N, Mayer C, Santic R, Navio RP, Wagner A, Bauer HC, Sperk G, Boehm U, Kofler B: Distribution of Alarin Immunoreactivity in the Mouse Brain. J Mol Neurosci 2012;46:18-32.

$>10$ Schrödl F, Trost A, Strohmaier C, Bogner B, Runge C, Kaser-Eichberger A, Eberhard N, Santic R, Kofler B, Reitsamer HA: Distribution of the regulatory peptide alarin in the eye of various species. Exp Eye Res 2013;106:74-81.

11 Van Der Kolk N, Madison FN, Mohr M, Eberhard N, Kofler B, Fraley GS: Alarin stimulates food intake in male rats and LH secretion in castrated male rats. Neuropeptides 2010;44:333-340.

12 Zhuang F, Zhou X, Gao X, Lou D, Bi X, Qin S, Sun C, Ye P, Wang Y, Ma T, Li M, Gu S: Cytokines and glucocorticoid receptors are associated with the antidepressant-like effect of alarin. Peptides 2016;76:115129.

13 Wada A, Wong PF, Hojo H, Hasegawa M, Ichinose A, Llanes R, Kubo Y, Senba M, Ichinose Y: Alarin but not its alternative-splicing form, GALP (Galanin-like peptide) has antimicrobial activity. Biochem Biophys Res Commun 2013;434:223-227.

14 Webling KE, Runesson J, Bartfai T, Langel U: Galanin receptors and ligands. Front Endocrinol (Lausanne) 2012;3:146.

15 Fraley GS, Leathley E, Lundy N, Chheng E, King I, Kofler B: Effects of alarin on food intake, body weight and luteinizing hormone secretion in male mice. Neuropeptides 2012;46:99-104. 


\section{Cellular Physiology Cell Physiol Biochem 2018;51:2041-2051

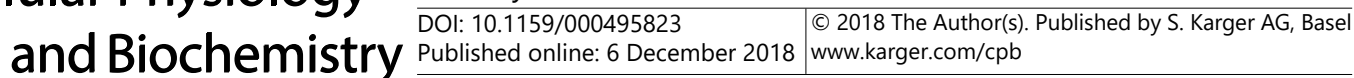

Fang et al.: Alarin and Metabolic Syndrome

16 Zhang Z, Wu Y, Sheng S, Guo L, He B, Fang P, Shi M, Bo P, Zhu Y: Intracerebroventricular Injection of Alarin Increased Glucose Uptake in Skeletal Muscle of Diabetic Rats. Plos One 2015;10:e0139327.

17 Guo L, Fang P, Yu M, Shi M, Bo P, Zhang Z: Central alarin ameliorated insulin resistance of adipocytes in type 2 diabetic rats. J Endocrinol 2014;223:217-225.

-18 Expert Panel on Detection, Evaluation, and Treatment of High Blood Cholesterol in Adults. Executive summary of the third report of the national cholesterol education program (NCEP) expert panel on detection, evaluation, and treatment of high blood cholesterol in adults (Adult Treatment Panel III). JAMA 2001;285:2486-2497.

19 Li K, Xu X, Hu W, Li M, Yang M, Wang Y, Luo Y, Zhang X, Liu H, Li L, Yang G: Glypican-4 is increased in human subjects with impaired glucose tolerance and decreased in patients with newly diagnosed type 2 diabetes. Acta Diabetol 2014;51:981-990.

20 Li M, Yang M, Zhou X, Fang X, Hu W, Zhu W, Wang C, Liu D, Li S, Liu H, Yang G, Li L: Elevated circulating levels of irisin and the effect of metformin treatment in women with polycystic ovary syndrome. J Clin Endocrinol Metab 2015;100:1485-1493.

-21 Matthews DR, Hosker JP, Rudenski AS, Naylor BA, Treacher DF, Turner RC: Homeostasis model assessment: insulin resistance and $\beta$-cell function from fasting plasma glucose and insulin concentrations in man. Diabetologia 1985;28:412-419.

22 Xu Q, Wang H, Li Y, Wang J, Lai Y, Gao L, Lei L, Yang G, Liao X, Fang X, Liu H, Li L: Plasma Sfrp5 levels correlate with determinants of the metabolic syndrome in Chinese adults. Diabetes Metab Res Rev 2017;33:e2896.

-23 Mikó A, Füredi N, Tenk J, Rostás I, Soós S, Solymár M, Székely M, Balaskó M, Brunner SM, Kofler B, Pétervári E: Acute central effects of alarin on the regulation on energy homeostasis. Neuropeptides 2017;64:117122.

24 Li L, Yang G, Shi S, Yang M, Liu H, Boden G: The adipose triglyceride lipase, adiponectin and visfatin are downregulated by tumor necrosis factor-alpha (TNF-alpha) in vivo. Cytokine 2009;45:12-19.

25 Yang G, Li L, Tang Y, Boden G: Short-term pioglitazone treatment prevents free fatty acid-induced hepatic insulin resistance in normal rats: Possible role of the resistin and adiponectin. Biochem Biophys Res Commun 2006;339:1190-1196.

-26 Norheim F, Langleite TM, Hjorth M, Holen T, Kielland A, Stadheim HK, Gulseth HL, Birkeland KI, Jensen J, Drevon CA: The effects of acute and chronic exercise on PGC- $1 \alpha$, irisin and browning of subcutaneous adipose tissue in humans. FEBS J 2014;281:739-749.

27 Chen G, Ye G, Zhang X, Liu X, Tu Y, Ye Z, Liu J, Guo Q, Wang Z, Wang L, Dong S, Fan Y: Metabolomics Reveals Protection of Resveratrol in Diet-Induced Metabolic Risk Factors in Abdominal Muscle. Cell Physiol Biochem 2018;45:1136-1148.

28 Pedersen BK, Febbraio MA: Muscles, exercise and obesity: skeletal muscle as a secretory organ. Nat Rev Endocrinol 2012;8:457-465. 